\title{
BM-MSCs promote prostate cancer progression via the conversion of normal fibroblasts to cancer-associated fibroblasts
}

\author{
SIMENG WEN ${ }^{1,2}$, YUANJIE NIU ${ }^{1}$, SHUYUAN YEH $^{2}$ and CHAWNSHANG CHANG ${ }^{2,3}$ \\ ${ }^{1}$ Chawnshang Chang Sex Hormone Research Center, Tianjin Institute of Urology, Tianjin Medical University, \\ Tianjin 300211, P.R. China; ${ }^{2}$ George Whipple Lab for Cancer Research, Departments of Pathology and Urology, \\ University of Rochester Medical Center, Rochester, NY 14642, USA; ${ }^{3}$ Sex Hormone Research Center, \\ Taichung Medical University. Taichung 404, Taiwan, R.O.C.
}

Received April 13, 2015; Accepted May 25, 2015

DOI: $10.3892 /$ ijo.2015.3060

\begin{abstract}
Recent studies have suggested that prostate cancer (PCa) is able to recruit bone marrow derived mesenchymal stem cells (BM-MSCs) to promote metastasis. The detailed mechanisms, especially the involvement of stromal cells, remain unclear. We found that the recruited BM-MSCs might be able to convert the normal fibroblasts to more cancer associated fibroblast (CAF)-like characteristics via alteration of secreted TGF $\beta-1$. The consequences of such conversion might then enhance the $\mathrm{PCa}$ growth and invasion. Addition of functional TGF $\beta-1$ or interruption with TGF $\beta-1$ inhibitor SB431542 led to alteration of the BM-MSCs-induced CAF conversion and influence on the PCa cell growth and invasion. Together, these results suggest that BM-MSCs not only can be directly recruited by PCa epithelial cells to promote PCa invasion, they can also go through conversion of normal fibroblasts to CAFs to enhance PCa cell growth and invasion. Targeting the infiltrating BM-MSCs via either interruption of the interaction between $\mathrm{PCa}$ and $\mathrm{BM}-\mathrm{MSC}$ or prevention of the conversion of NFs to CAFs via inhibition of TGF $\beta-1$ signal may result in the suppression of PCa progression.
\end{abstract}

\section{Introduction}

The majority of identified tumors, including prostate cancer (PCa) were proven to be originated from epithelial cells (1). For many years, tumorigenesis has been classically regarded

Correspondence to: Dr Yuanjie Niu, Tianjin Institute of Urology, Tianjin Medical University, Tianjin 300211, P.R. China

E-mail: niuyuanjie@gmail.com

Professor Chawnshang Chang, Department of Pathology, University of Rochester Medical Center, Rochester, NY 14642, USA

E-mail: chang@urmc.rochester.edu

Key words: bone marrow derived mesenchymal stem cell, cancer associated fibroblast, prostate cancer, TGF $\beta-1$ as a largely cell-autonomous process involving genetically transformed cancer cells. Consequently, the focus of cancer research has been on genetic changes in cancer cells, with the progression from normal to malignant state. Recently, increasing number of studies suggest that the progression of tumors might not only rely on the cell-autonomous process of cancer cells themselves but tumors could be also influenced by the surrounding cells (2). The tumor microenvironment (TME) participation in tumor is now well accepted. Most of the solid tumors are surrounded by complicated microenvironment, including the extracellular matrix (ECM), different stromal cells (smooth muscle cells and fibroblasts), other resident cells and recruited inflammatory cells or bone marrow mesenchymal stem cells (BM-MSCs) (2-4).

The peritumoral fibroblasts are considered as active fibroblasts, which are also known as cancer associated fibroblasts (CAFs) or myofibroblasts. In the normal tissue, fibroblasts can be activated for wound healing or fibrosis (5). The activated myofibroblasts can gain contractile stress fibers by expressing $\alpha$-smooth muscle actin ( $\alpha$-SMA) (6) that allows them to gain better mobility via stronger contractile and secretory capabilities. Therefore, more and more data suggest that myofibroblasts or CAFs might have the capacity to promote tumor growth and metastasis, either via direct interaction with tumor epithelial cells or via the recruitment of inflammatory cells $(7,8)$.

Recent studies found PCa cells, but not normal prostate cells, could better recruit BM-MSCs and the consequences of such increased recruitment might then lead to enhanced PCa metastasis (9). The involvement of stromal cells in such enhanced PCa metastasis, however, remains unclear. In this study, we focused on the roles of stromal cells in the BM-MSCs capacity to enhance PCa growth and invasion and results revealed that recruited BM-MSCs converted normal fibroblasts (NFs) to more CAF-like cells to further promote PCa cell growth and invasion.

\section{Materials and methods}

Cell culture. TRAMP-C1, CWR22Rv1, LNCaP, and C4-2 cell lines were obtained from ATCC (Manassas, VA, USA). 
TRAMP-C1 cells were maintained in Dulbecco's modified Eagle's medium with 10\% FBS. CWR22Rv1, LNCaP, and C4-2 cells were cultured in RPMI-1640 medium with 10\% FBS.

Mouse primary BM-MSCs were isolated from wild-type C57BL/6 mice (Jackson Laboratory, Bar Harbor, ME, USA) as previouly described (10). Tibias and femurs were dissected from 6-week-old mice. After bones were cut, the marrows were flushed out with $5 \mathrm{ml}$ DMEM by using a needle and syringe, and re-suspended in DMEM plus 15\% FBS. After filtration, mononucleated cells were maintained in DMEM (Gibco) with $15 \%$ FBS, 2 mM L-glutamine, $100 \mathrm{U} / \mathrm{ml}$ penicillin, $100 \mathrm{mg} / \mathrm{ml}$ streptomycin, and $10 \mathrm{mM}$ HEPES. All of the cells were incubated at $37^{\circ} \mathrm{C}$ with $5 \%$ humidified $\mathrm{CO}_{2}$ for $24 \mathrm{~h}$, then the non-adherent cells were removed by replacing the medium every 3 days for $\sim 1$ week. When the cells grew to confluence, they were harvested with $0.25 \%$ trypsin and $1 \mathrm{mM}$ EDTA (Hyclone) for experiments.

Human BM-MSCs were purchased from Stem Cell Technologies (Vancouver, BC, Canada), and maintained in Human MesenCult ${ }^{\circledR}$ Proliferation kit (Stem cell Technologies Inc.). BM-MSCs were already verified by adipogenesis and osteogenesis differentiations in our lab (10). As described previously (11), mouse CAFs was isolated from the prostate stromal region of transgenic adenocarcinoma of the prostate mice in our lab. Mouse NFs were isolated from the prostate stromal region of benign prostate mice in the same way. pshTERT is an immortalized stromal cell line, stably expressing the human telomerase catalytic subunit-hTert (a kind gift from the New York University, named pshTERT). NFs and CAFs were cultured in RPMI-1640 medium. All cells were maintained in a humidified $5 \% \mathrm{CO}_{2}$ environment at $37^{\circ} \mathrm{C}$.

Cell viability assay (MTT). TRAMP-C1 cells $\left(10^{4}\right)$ with different treatment were seeded in 24-well plates, and cultured in 10\% FBS media for 0, 2, 4 and 6 days. Cells from different time-points were harvested, then we added 3-(4,5-dimethylthiazol-2-yl)-2,5-diphenyltetrazolium bromide (MTT, $0.5 \mathrm{mg} / \mathrm{ml}$ in normal media), incubated for $2 \mathrm{~h}$ and removed the media. The cellular staining products were dissolved in $0.5 \mathrm{ml} \mathrm{DMSO}$. The absorbance at OD570 was measured by spectrophotometry (Beckman Du640B). The data represent means \pm SD from three independent wells and confirmed by three independent experiments.

BrdU labeling. Cells $\left(10^{3}\right)$ were seeded on the 6-well plates, given certain treatment (such as NFs or $\mathrm{CAFs}$ ) and cultured to $50 \%$ confluence. BrdU labeling reagent (Invitrogen) was added to the cultured cells for 1:100 dilution in the culture media for $24 \mathrm{~h}$. After labeling, staining was performed according to the manual instructions (Invitrogen, BrdU staining kit). The data represent means \pm SD from three independent wells and confirmed by three independent experiments.

Invasion assay. The 6-well transwells (Corning, \#3422) were coated with growth factor reduced matrix gel diluted with serum-free RPMI-1640 media (1:5 diluted), and dried overnight at $37^{\circ} \mathrm{C}$. Cells $\left(10^{5}\right)$ after different treatments were re-suspended with serum-free media and seeded in the upper transwells. Media with 10\% FBS was put in the lower wells. After incubation for $48 \mathrm{~h}$, the cells on the upper surface of the transwells were removed with cotton swabs. Cells on lower filter surfaces were fixed with $75 \%$ ethanol, stained with $0.1 \%$ toluidine blue in PBS. Invaded cells were counted under a microscope. The data represent means \pm SD from three independent wells and confirmed by three independent experiments.

Western blot analysis. Harvested cells were washed with PBS twice and lysed in RIPA buffer $(50 \mathrm{mM}$ Tris- $\mathrm{HCl} / \mathrm{pH} 7.4$, $1 \% \mathrm{NP}-40,150 \mathrm{mM} \mathrm{NaCl}, 1 \mathrm{mM}$ EDTA, $1 \mathrm{mM} \mathrm{Na}_{3} \mathrm{VO}_{4}, 1 \mathrm{mM}$ $\mathrm{NaF}, 1 \mathrm{mM}$ okadaic acid and $1 \mathrm{mg} / \mathrm{ml}$ aprotinin, leupeptin, and pepstatin) with proteinase inhibitor. Samples (30 $\mu$ g protein) were loaded on $10 \%$ SDS-PAGE gel to be separated and transferred to PVDF membranes at $4^{\circ} \mathrm{C}(100 \mathrm{~V}, 90 \mathrm{~min})$. Membranes were blocked in 5\% fat-free milk with 3\% BSA in PBST for $1 \mathrm{~h}$ at room temperature, and incubated with appropriate dilutions of primary antibodies ( $\alpha$-SMA, Abcam \#ab7817, 1:300; GAPDH, Santa Cruz \#sc-32233, 1:3,000) overnight at $4^{\circ} \mathrm{C}$. Then the membranes were washed, and incubated with HRP conjugated anti-mouse IgG for $1 \mathrm{~h}$ at room temperature. The blots were developed in ECL mixture and visualized by an imager.

RNA isolation and quantitative analysis. Total cellular RNAs were extracted by TRIzol reagent (Invitrogen) according to the manufacturer's instructions. Total RNA (1 ng) was used to synthesize first-strand complementary DNA by reverse transcriptase Superscript III (Invitrogen). The amount of certain cDNA was measured in real-time PCR assays using a SYBR green Bio-Rad CFX96 system. Primers were: mouse $\alpha$-SMA: sense 5'-CCCAGACATCAGGGAGTAATGG-3', antisense 5'-TCTATCGGATACTTCAGCGTCA-3'; mouse CD90: sense 5'-TGCTCTCAGTCTTGCAGGTG-3', antisense 5'-TGGAT GGAGTTATCCTTGGTGTT-3'; mouse IGF-1: sense 5'-CAC ATCATGTCGTCTTCACACC-3', antisense 5'-GGAAGCAA CACTCATCCACAATG-3'; mouse IGF-2: sense 5'-GTGCTG CATCGCTGCTTAC-3', antisense 5'-CGGTCCGAACAGAC AAACT-3'; mouse PDGFA: sense 5'-TGGCTCGAAGTCAG ATCCACA-3', antisense 5'-TTCTCGGGCACATGGTTA ATG-3'; mouse TGF $\beta$-1: sense 5'-CCACCTGCAAGACCATG GAC-3', antisense 5'-CTGGCGAGCCTTAGTTTGGAC-3'; mouse GM-CSF: sense 5'-GGCCTTGGAAGCATGTAGA GG-3', antisense 5'-GGAGAACTCGTTAGAGACGACTT-3'; human IGF-1: sense 5'-ATGCTCTTCAGTTCGTGTGTG-3', antisense 5'-GCACTCCCTCTACTTGCGTTC-3'; human IGF-2: sense 5'-GTGGCATCGTTGAGGAGTG-3', antisense 5'-CACGTCCCTCTCGGACTTG-3'; human TGF $\beta$-1: sense 5'-GGCCAGATCCTGTCCAAGC-3', antisense 5'-GTGGGT TTCCACCATTAGCAC-3'; human GM-CSF: sense 5'-GGG AGCATGTGAATGCCATC-3', antisense 5'-GCAGTGTCTCT ACTCAGGTTCAG-3'. The expression level of the other genes was normalized to the expression of mouse GAPDH (sense 5'-AGGTCGGTGTGAACGGATTTG-3', antisense 5'-TGTAG ACCATGTAGTTGAGGTCA-3') or human GAPDH (sense 5'-TGGCTTCATAGGTGACTTCCA-3', antisense 5'-AAGG ACCTGTCTAGGTTTGATGC-3').

Immunofluorescence (IF) staining. NFs with different treatments were seeded on 4-well chamber slides and fixed with $4 \%$ paraformaldehyde in PBS for $1 \mathrm{~h}$ at $15-25^{\circ} \mathrm{C}$, washed with 
A

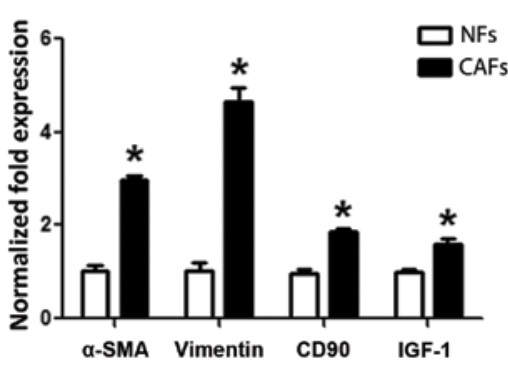

C

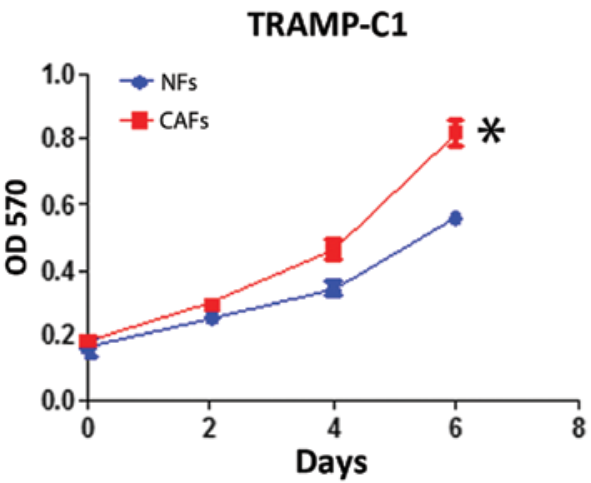

B

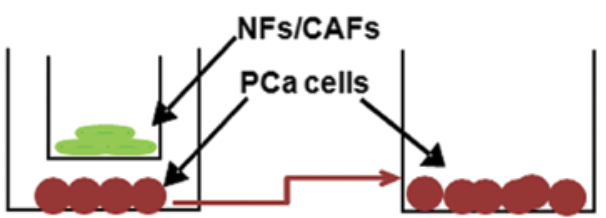

1.Co-culture NFs/CAFs with PCa cells
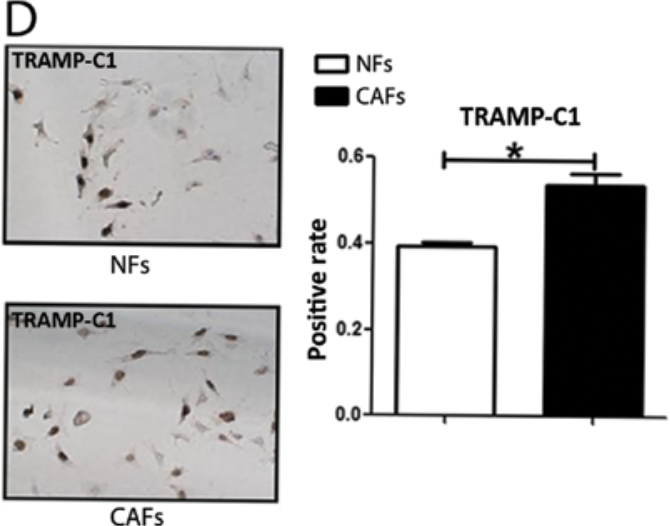

$\mathrm{E}$

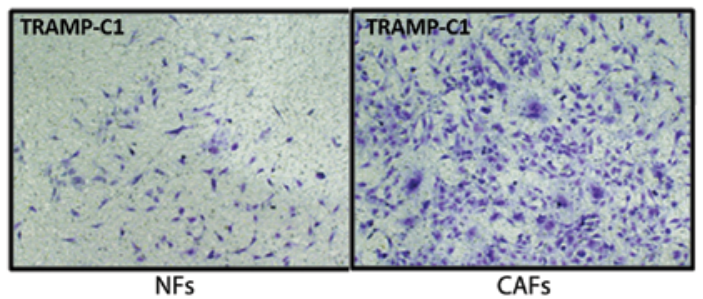

TRAMP-C1

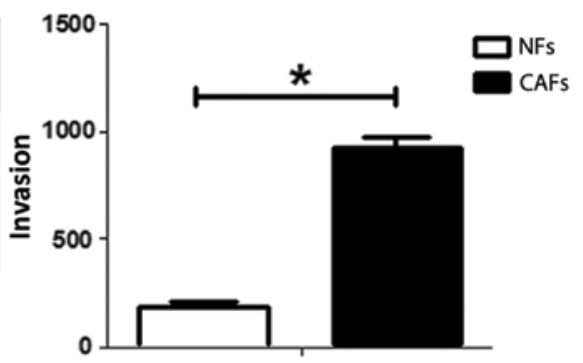

Figure 1. Identity of normal fibroblasts (NFs) and cancer associated fibroblasts (CAFs) isolated from the tumor bearing TRAMP mice. Characteristics of NFs and CAFs. NFs and CAFs were isolated from normal and cancer tissues and immortalized using SV40-T antigen incorporation. (A) Real-time PCR results show the obtained NFs and CAFs differentially express CAF specific markers, including CD90, IGF-1 and $\alpha$-SMA. (B) Carton shows our protocols to do the growth and invasion assays. (C) The capabilities of NFs and CAFs to promote PCa cell growth are different in MTT assays. (D) BrdU staining shows the capability difference of NFs and CAFs to promote PCa cell growth. (E) The capabilities of NFs and CAFs to promote PCa cells invasion are different in transwell assays. ${ }^{*} \mathrm{p}<0.05$.

PBS. Then slides were incubated in permeabilisation solution ( $0.5 \%$ Triton X-100 in PBS) for $5 \mathrm{~min}$. Following blockage with $5 \%$ BSA for $1 \mathrm{~h}$ samples were incubated with primary antibody (anti-mouse $\alpha$-SMA antibody, Abcam \#ab7817, $1: 100)$ at $4^{\circ} \mathrm{C}$ overnight. After washing in $1 \mathrm{X}$ PBS, incubated with 1:200 diluted fluorescent secondary antibody for IF (Alexa 488 tagged). Signals were observed under fluorescence microscope.

In vivo tumor studies. Male 6- to 8-week-old nude mice were used. Luciferase expressing CWR22Rv1 cells $\left(1 \times 10^{6}\right)$, stably transfected by pCDNA3.0-luciferase plasmid, were mixed with Matrigel (1:1 in volume) and injected to the anterior lobes of 5 nude mice as control group. Eight mice were injected with CWR22Rv1 cells together with parental pshTERT cells (1:1). And another 8 mice were injected with CWR22Rv1 cells together with BM-MSCs pre-treated pshTERT cells. Six weeks after injection, mice were sacrificed and tumors were weighed. The research was approved and conducted following the rules and regulations of the University Committee of Animal Research (UCAR), No. 2002-296), which was fully credited by Association for Assessment and Accreditation of Laboratory Animal Care (AAALAC, No. A-3292-01), at the University of Rochester Medical Center.

Statistical analysis. Values were expressed as mean \pm standard deviation (SD). The Student's t- and ANOVA tests were used to calculate p-values. p-values were two-sided, and considered statistically significant when $<0.05$.

\section{Results}

Identification of NFs and CAFs isolated from the PCa bearing mice. We first confirmed NFs and CAFs that were isolated 


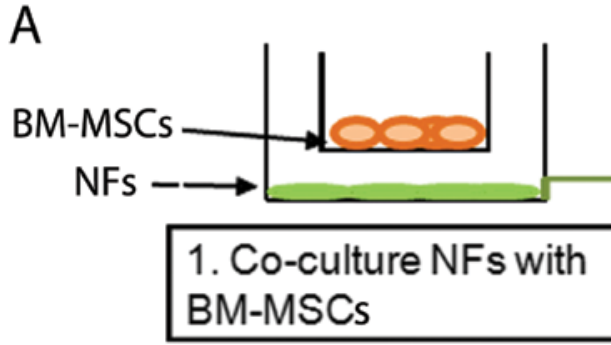

B

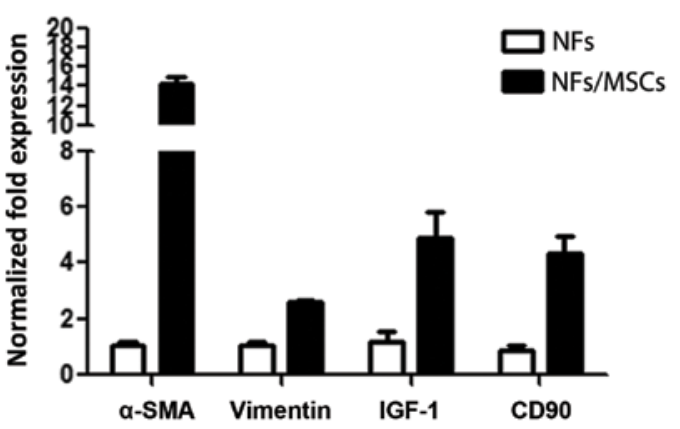

\section{Extract Total RNA or protein from these NFs}

C

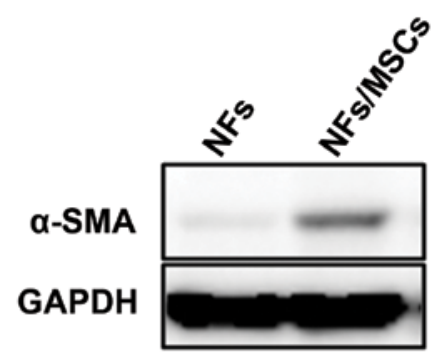

Figure 2. Co-culture of BM-MSCs and NFs changes the characteristics of NFs. The NF characteristics were altered when co-cultured with the BM-MSCs. NFs were co-cultured, with or without mouse primary BM-MSCs, which had been isolated from the normal B6 mice and cultured in transwell plates for 7 days. (A) The experimental protocol. (B) Total RNAs were then obtained from the NFs. The expression of CAF markers in NFs treated by BM-MSCs (MSCs) conditional media and control regular media were analyzed by real-time PCR. (C) Total proteins were extracted from NFs (BM-MSCs pretreated and control media-treated) for western blot analysis.

A

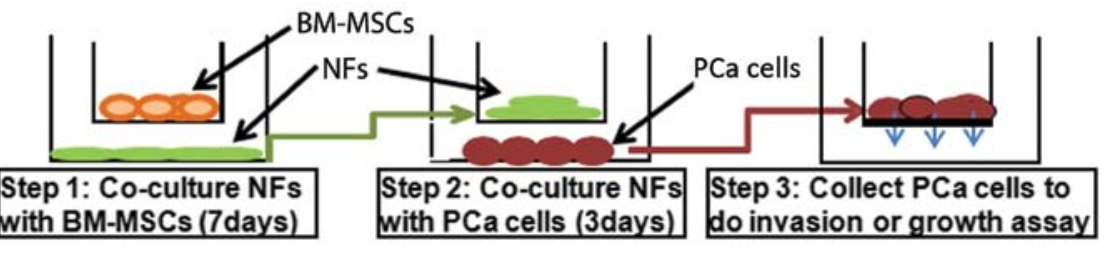

B
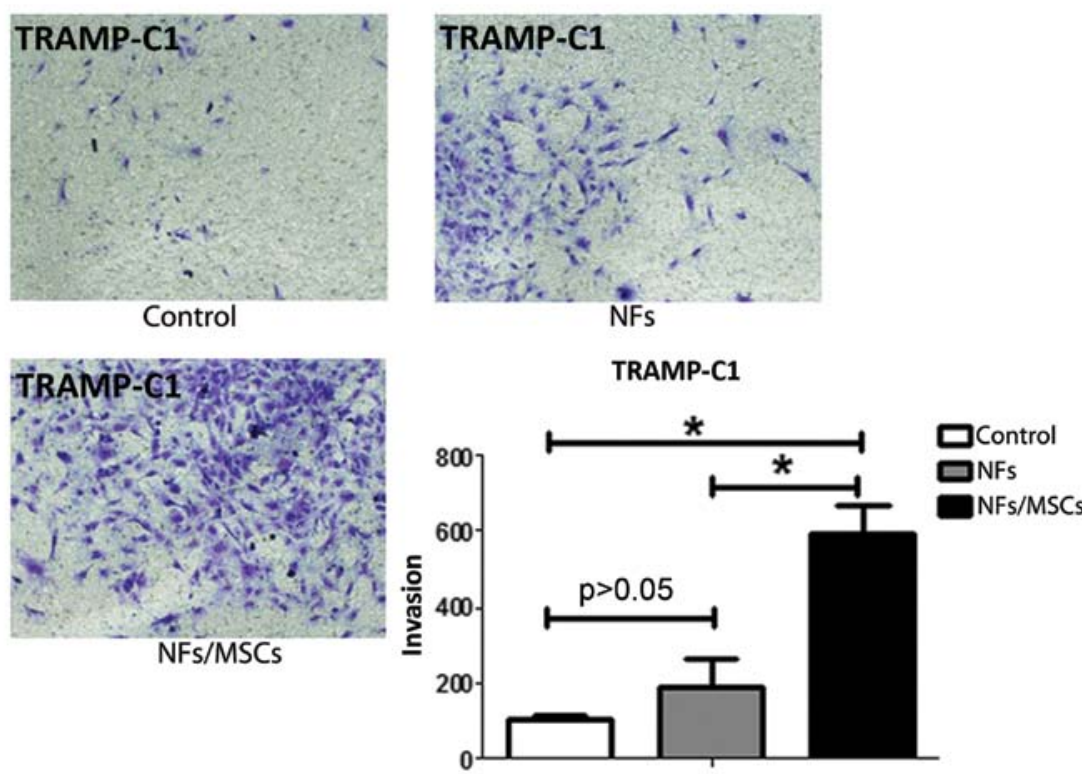

Figure 3. NFs promote PCa cell invasion when pre-treated with BM-MSCs. (A) The strategy of performing PCa cell invasion test. NFs (mouse NF or human pshTERT cells) were co-cultured with mouse or human BM-MSCs for 7 days, respectively. The NFs were subsequently incubated with PCa cells for 3 days, and then the PCa cells were harvested, and their invasion ability was tested in transwell plates. (B) The invasion ability of TRAMP-C1 cells co-cultured with NFs or BM-MSCs (MSCs)-treated NFs were tested. Quantitation shown on the right. "p $<0.05$. 
A

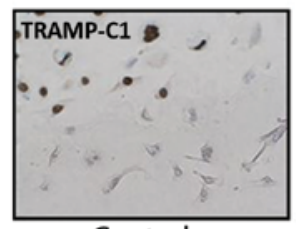

Control

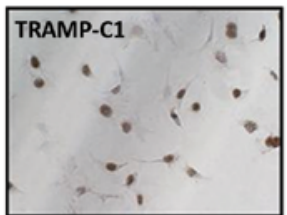

NFs/MSCs

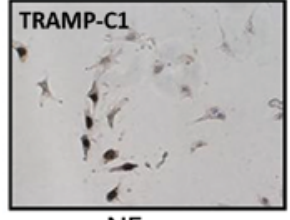

$\mathrm{NFs}$

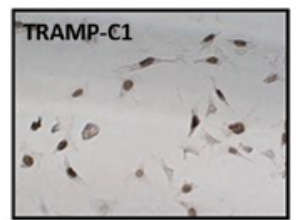

CAFs
TRAMP-C1

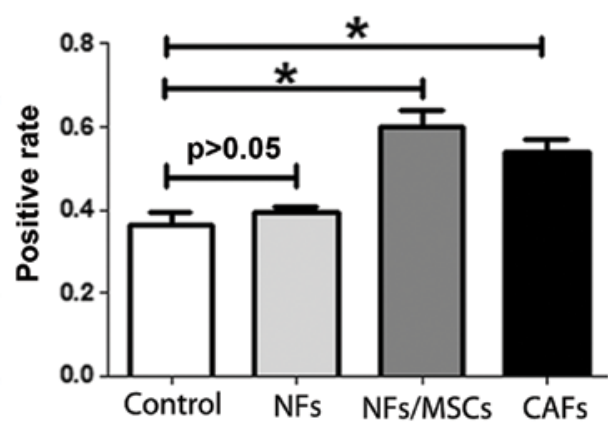

B

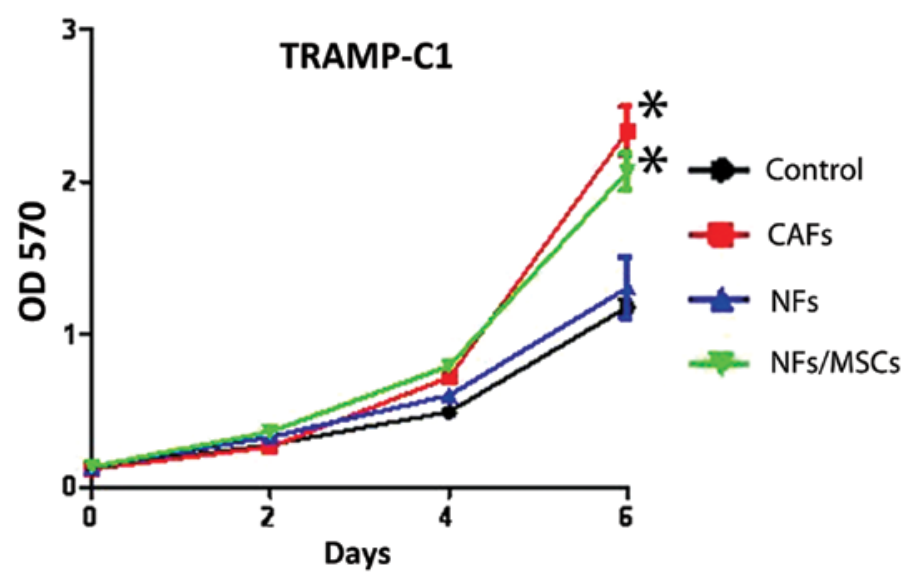

Figure 4. The BM-MSC pre-treated NFs promote PCa cells growth. Growth assay follows the protocol demonstrated in Fig. 3A, similar to invasion assay. NFs were co-cultured with the primary mouse BM-MSCs for 7 days, subsequently incubated with PCa cells for 3 days, which were harvested for the growth assay. (A) The growth of TRAMP-C1 cells co-cultured with NFs, BM-MSCs pre-treated NFs, CAFs and control media was demonstrated by BrdU staining, the quantitation of growth rates are shown on the right. (B) The viability of TRAMP-C1 cells in different treatments were tested in MTT assay. Quantitation is shown on the right. p $<0.05$.

from TRAMP mice with several markers for CAFs. The co-expression of $\alpha$-SMA and vimentin is commonly used to define CAFs (12). CD90 (8) and IGF-1 (13) were also reported highly expressed in CAFs. We found the expression of $\alpha$-SMA, vimentin, CD90 and IGF-1 in CAFs were higher in CAFs than NFs with qPCR (Fig. 1A), western blot analysis (Fig. 5D) and IF assays (Fig. 5E). We further compared their ability to enhance $\mathrm{PCa}$ cell growth and invasion as early reports documented that CAFs have much better capacity than NFs to enhance PCa cell growth and invasion (14). We co-cultured PCa epithelial cells with NFs vs CAFs in the co-culture systems (Fig. 1B), and results from MTT (Fig. 1C) and BrdU staining (Fig. 1D) assays revealed that the CAFs have better capacity than NFs to enhance PCa cells growth and proliferation. Furthermore, the results from the transwell invasion assay also showed that the CAFs had much better capacity than NFs to enhance PCa cell invasion (Fig. 1E).

Together, results from Fig. 1 confirmed the CAFs and NFs used in these studies are correct by showing CAFs have better capacity than NFs to enhance PCa cell growth and invasion.
Molecular characteristics of NFs are changed upon co-culture with BM-MSCs. To investigate whether BM-MSCs have any influence on the conversion of prostate NFs to CAF cells, we co-cultured BM-MSCs with NFs (Fig. 2A) at a ratio of 1:10 for 7 days, and results from qPCR and western blot analyses revealed increased CAF markers, including $\alpha$-SMA, CD90, and IGF-1 in NFs upon co-culture with BM-MSCs (Fig. 2B and C). Similar results were obtained when we replaced the ratio of BM-MSCs with NFs from 1:1 to 1:2 or 1:4 (data not shown). These results suggest that BM-MSCs can influence the conversion of NFs to cells with characteristics more close to CAFs.

NFs promote $P C a$ cell invasion when pre-treated with $B M-M S C s$. One of the key phenotypes of CAFs is their capability to promote tumor metastasis (Fig. 1E) (2). Our next experiment was to test whether BM-MSCs-treated NFs also would have a similar capability. After co-culturing mouse BM-MSCs and mouse NFs for 7 days, we removed the mouse BM-MSCs (to eliminate the BM-MSCs effect on PCa cells) and collected mouse NFs. These pre-treated NFs were subse- 
A
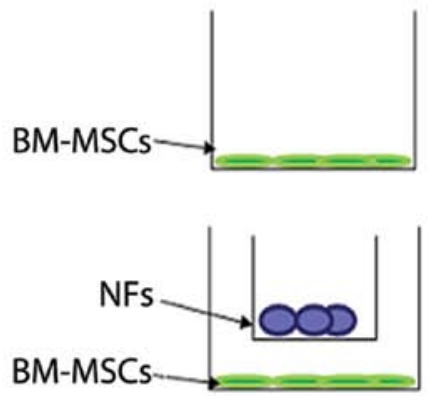

B

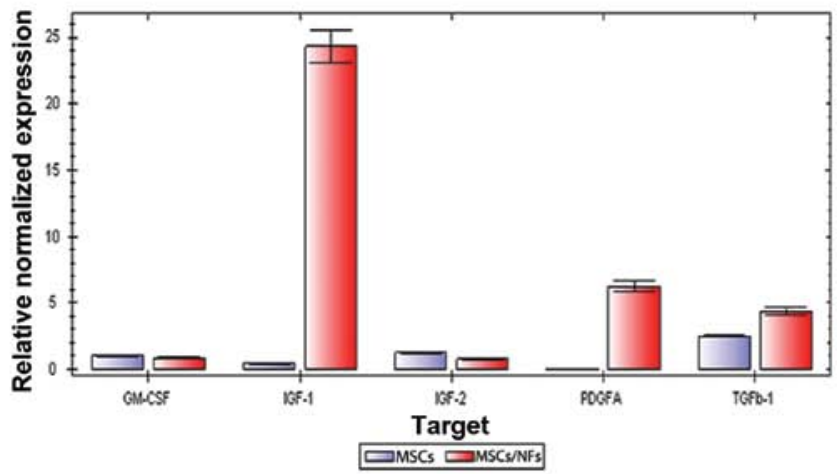

D
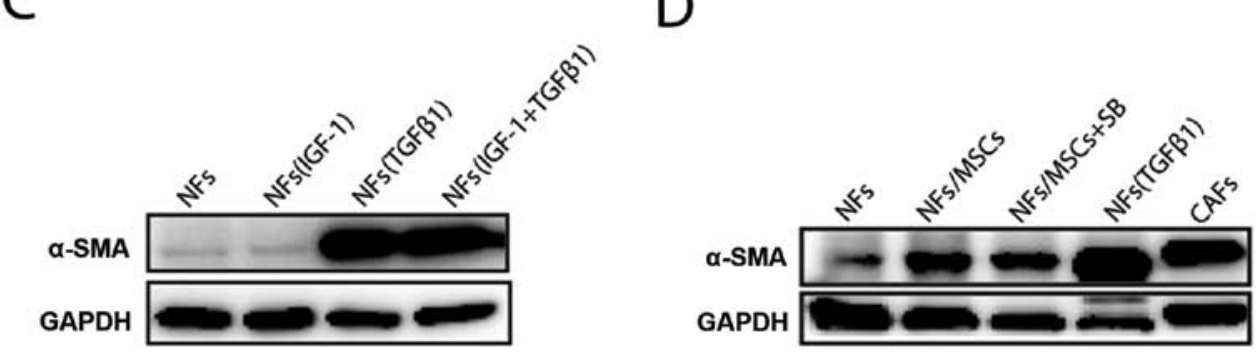

$\mathrm{E}$

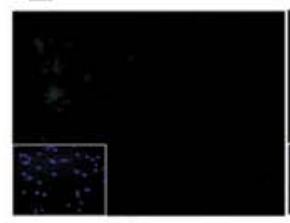

NFs

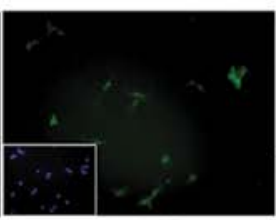

NFs/MSCs

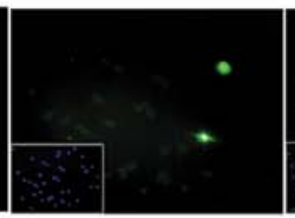

$\mathrm{NF} s / \mathrm{MSC}+\mathrm{SB}$

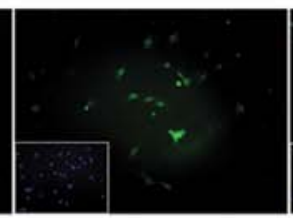

$\mathrm{NFs}(\mathrm{TGF} \beta 1)$

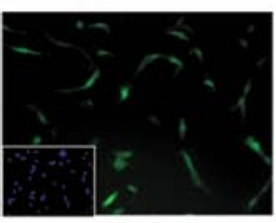

CAFs

$\mathrm{F}$

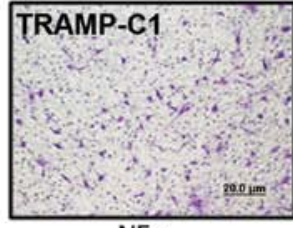

NFs

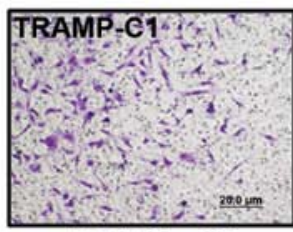

$\mathrm{NFs} / \mathrm{MSCs}+\mathrm{SB}$

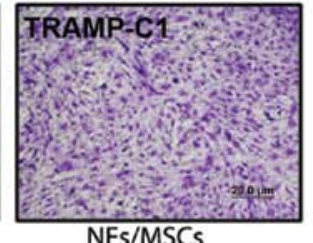

$\mathrm{NFs} / \mathrm{MSCs}$

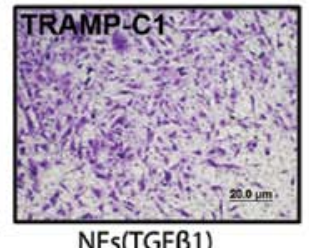

TRAMP-C1

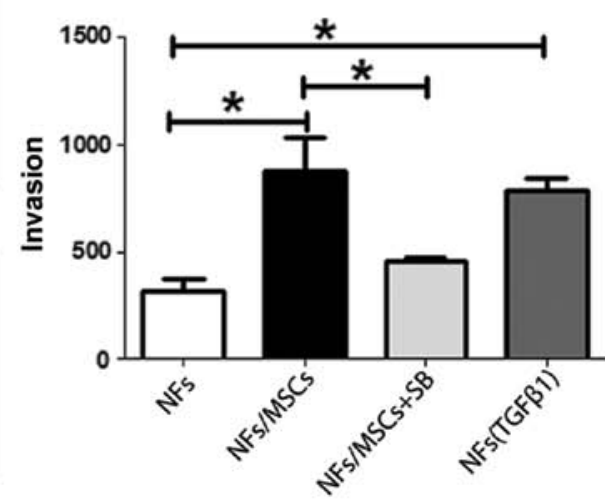

Figure 5. Mechanism by which BM-MSCs alter the NF characteristics. (A) The strategy of extracting total RNA from BM-MSCs after co-culture with NFs. or BM-MSCs alone, which were used as a control. (B) Real-time PCR assay shows TGF- $\beta 1$, IGF-1 and PDGFA mRNA expression levels are increased in mouse BM-MSCs upon co-cultured with mouse NFs. (C) For western blot assay, NF cells were treated with TGF- $\beta 1$ or (and) IGF-1 for 3 days, then total protein from NFs was extracted. The protein expression of $\alpha$-SMA in NFs, the major marker of CAF, was increased after treated with TGF- $\beta 1$ but not IGF-1. (D) When inhibitor of TGF- $\beta 1$ was added into the co-culture system, $\alpha$-SMA was lightly decreased, showing the NFs to CAFs conversion was partially blocked (E) Immunofluorescence results showing the expression of $\alpha$-SMA after different treatments. (F) Co-cultured TRAMP-C1 cells with differently pre-treated NFs. The TRAMP-C1 cells were collected for the invasion assay. Quantitation is shown on the right. "p $<0.05$.

quently incubated with the mouse PCa epithelial TRAMP-C1 cells (at the ratio of 1:1) for 3 days (see detailed procedure in Fig. 3A). The results showed that NFs pre-treated/incubated with BM-MSCs have the capacity to enhance the PCa cell invasion (Fig. 3B).
Similar results were obtained when we replaced mouse BM-MSCs and mouse NFs with human BM-MSCs and human stromal pshTERT cells showing pshTERT cells (after co-cultured with human BM-MSCs) were able to promote $\mathrm{PCa}$ C4-2 (Fig. 1) and LNCaP cells invasion (Fig. 6B). 
A
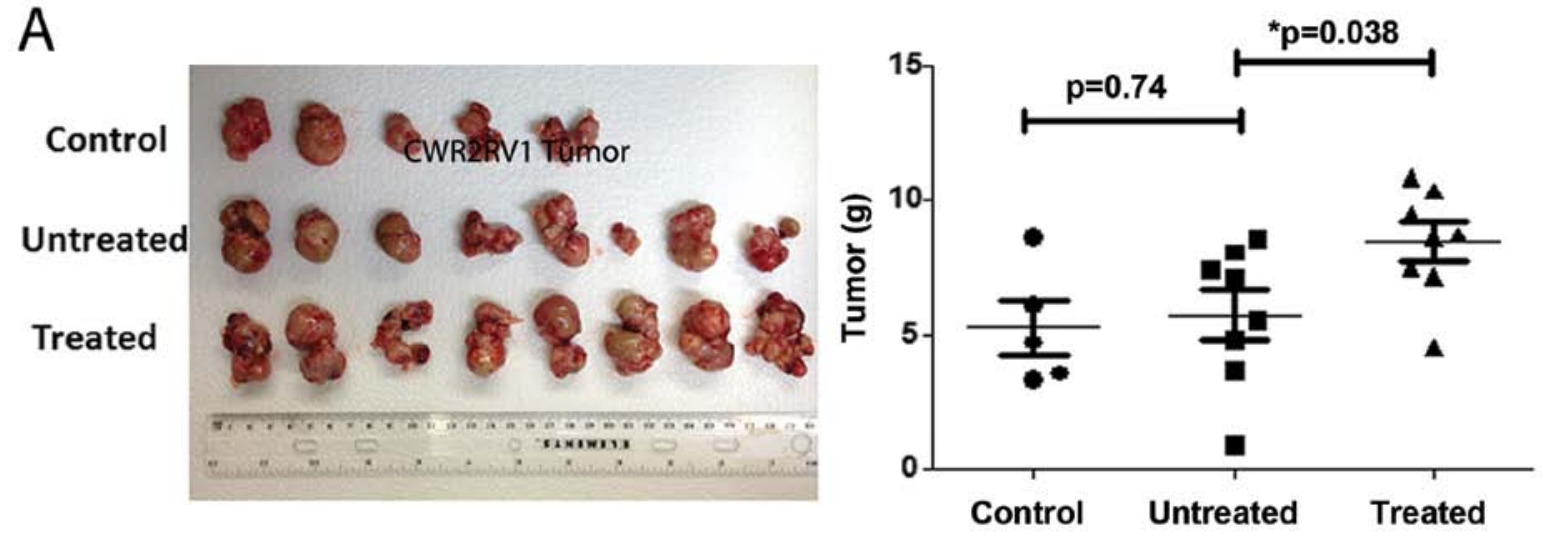

B

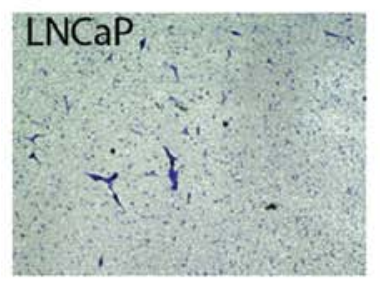

Control

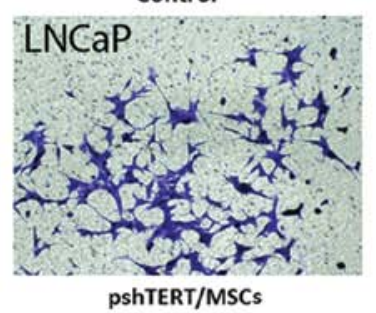

C

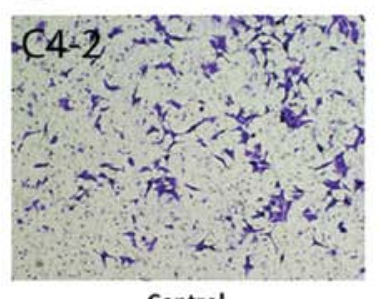

Control

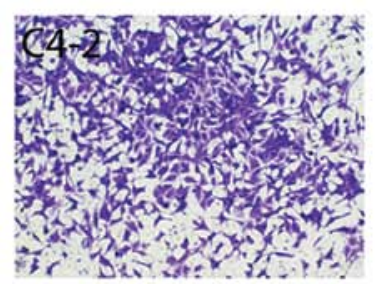

pshTERT/MSCs

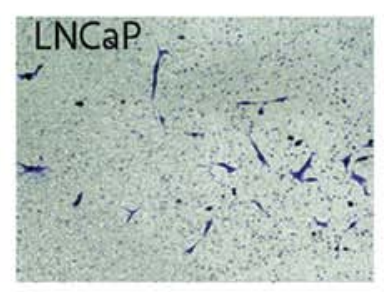

pshTERT
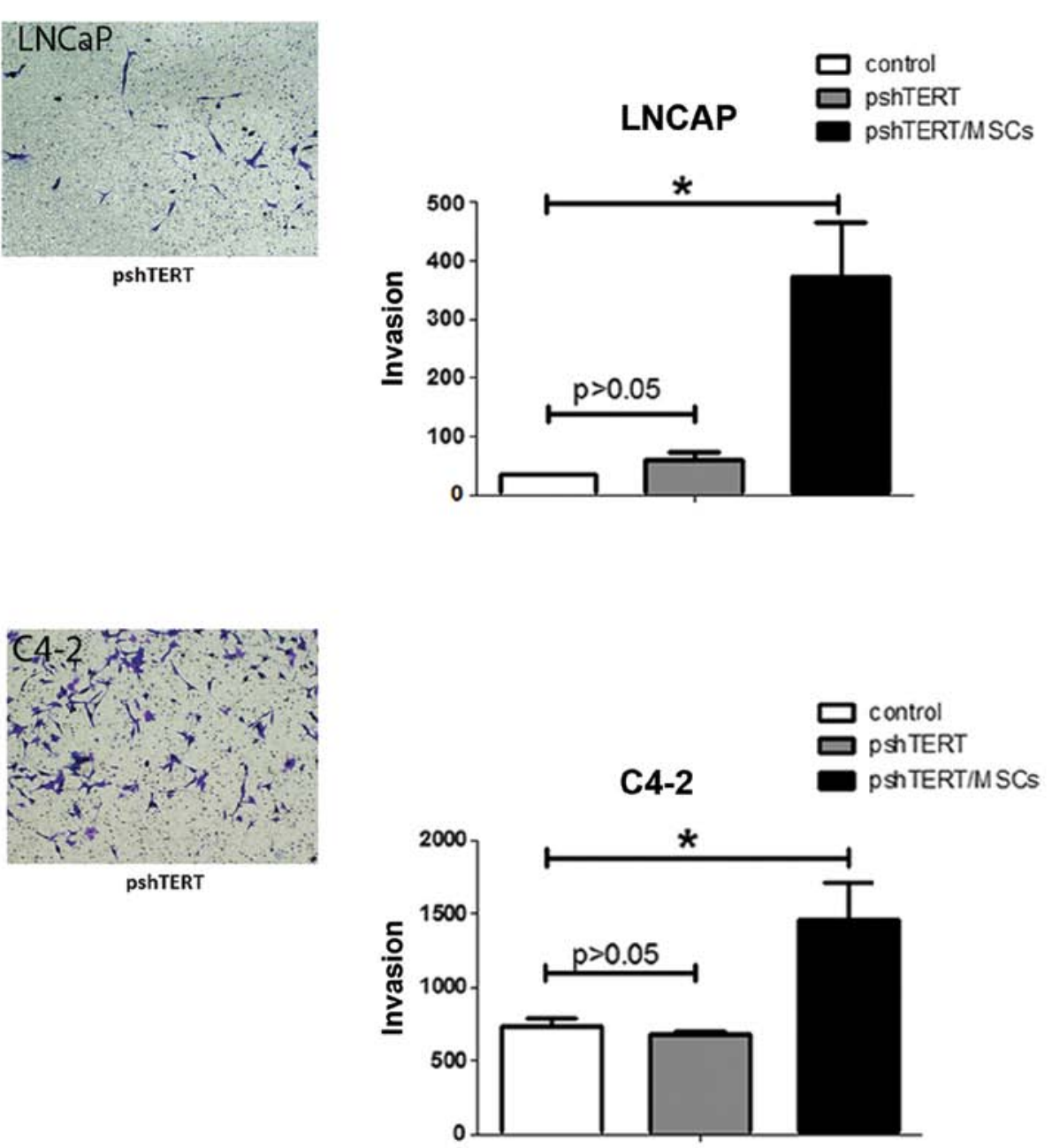

Figure 6. The BM-MSCs pre-treated NFs promote human PCa tumor growth and PCa cell invasion. (A) CWR22Rv1 cells, either alone or together with untreated or BM-MSCs 7-day pre-treated pshTERT cells were orthotopically injected into nude mice. Mouse group 1, CWR22Rv1 alone; group 2, CWR22Rv1 cells + pshTERT cells (media treated, control); group 3, CWR22R1 + pshTERT cells (pre-treated with hBM-MSCs for 7 days). For groups 2 and 3, the mixtures of CWR22Rv1 and pshTERT cells in a ratio of 5:1 were used in injection. After 6 weeks, mice were sacrificed and tumor weight was analyzed. Quantitation is shown on the right. " $\mathrm{p}<0.05$. (B) Images of the invasion ability of LNCaP cells co-cultured with pshTERT or human BM-MSCs treated pshTERT cells. (C) Images of the invasion ability of C4-2 cells co-cultured with pshTERT or human BM-MSCs treated pshTERT cells.

The above, together with the results in Fig. 3 suggest that the prostate NFs may acquire CAFs characteristics via co-culture with BM-MSCs to enhance PCa cell invasion.

NFs promote PCa cell growth when pre-treated with $B M-M S C s$. Another key phenotype of CAFs is their capability to promote tumor growth (2). Using BrdU staining assay and MTT assay to detect PCa cells proliferation (Fig. 4A) and growth (Fig. 4B), we found that the NF pre-treated BM-MSCs increased the PCa cells growth (Fig. 4A) and proliferation (Fig. 4B). In vivo mouse studies using mice with orthotopically xenografted CWR22Rv1 cells co-implanted with NF 
cells pre-treated with either BM-MSCs or control media for 7 days also showed that NFs pre-treated with BM-MSCs have larger tumor size as compared to the media control (Fig. 6A).

The in vitro and in vivo results from Figs. 4 and 6A suggest that the prostate NFs may acquire the CAF characteristics via co-culture with BM-MSCs to enhance PCa cell growth and proliferation.

BM-MSCs secrete TFG $\beta$-1 to promote the conversion from $N F$ s to CAFs. Recent studies demonstrated that some cytokines or growth factors including GM-CSF, IGF-1, IGF-2, PDGFA and TFG $\beta-1$ induced the conversion of NFs to CAFs (15-17). To investigate which growth factors or cytokines secreted by BM-MSCs might induce this transformation, we extracted RNAs from BM-MSCs, with or without co-culture with NFs (Fig. 5A), to assay their differential expression of these growth factors/cytokines. The qPCR analyses revealed that expression of PDGFa, IGF-1 and TGF $\beta-1$ in BM-MSCs was increased after co-culture with NFs (Fig. 5B). Similar results with increased IGF-1 and TGF $\beta-1$ also occurred when we replaced mouse BM-MSCs/NFs cells with human BM-MSCs/NFs cells PCa (data not shown).

We then applied two different approaches to further confirm these results: first we added IGF-1 and TGF $\beta-1$ recombinant proteins to NFs to mimic the BM-MSCs effect and found only TGF $\beta-1$ induced expression of $\alpha$-SMA (Fig. 5C). We then used an interruption approach via addition of TGF $\beta-1$ inhibitor SB431542 to the co-cultured NFs and BM-MSCs and found slightly suppressed $\alpha$-SMA expression (Fig. 5D and E). Importantly, addition of the TGF $\beta-1$ inhibitor SB431542 suppressed the BM-MSCs/NFs induced PCa cells invasion (Fig. 5F).

Results in Fig. 5 suggest that BM-MSCs may be able to trigger the conversion of NFs to CAFs via altering the secretion of TGF $\beta-1$.

\section{Discussion}

PCa is the most common malignant tumor of men in the USA with the second highest death rate (1). Even though the current standard therapy of androgen deprivation therapy (ADT) for the later stage $\mathrm{PCa}$ is effective in the beginning, eventually the PCa still progresses into the castration resistant stage with metastasis (18). Several hypotheses were used to explain the mechanisms by which PCa could escape from ADT (19), yet none of them was applied successfully to cure PCa. It is now widely recognized that $\mathrm{PCa}$, like other tumors, is regulated by multiple signaling from multiple cells existing in the TME, including luminal epithelial cells (19), epithelial basal cells, stromal cells, stem/progenital cells (20), BM-MSCs (9), endothelial cells (21), and macrophages (22).

The stromal CAFs in TME, were also reported to be able to influence the PCa progression (2). However, the origin of CAFs remained unclear. Early studies suggested that the most important source of CAFs could come from the resident NFs (17), especially the spindle-like fibroblasts surrounding tumors (so-called peritumor fibroblasts), and not the fibroblasts far away from tumor sites, with distinct characteristics of highly expressed $\alpha$-SMA, CD90, IGF-1 $(8,23,24)$ that can promote tumor growth and invasion $(7,14)$. The second source of CAFs could come from the endothelial-mesenchymal transition (EndMT) (25) with increased mesenchymal markers of fibroblast-specific protein-1 (FSP1) and decreased CD31/PECAM (25). Importantly, BM-MSCs were reported to be the potential third source of CAFs (26) that might contribute $25 \%$ of the CAF population (27).

The bone marrow is a complex tissue containing hematopoietic stem/progenitor cells and their connective tissue mesenchymal cells constituting the bone marrow microenvironment. BM-MSCs comprise multifunctional non-hematopoietic stem cells that have differentiating capabilities (28). In mouse models, BM-MSCs were demonstrated to be able to migrate and incorporate into other tissues where BM-MSCs were able to elicit tissue-specific differentiation. For example, BM-MSCs contributed to tissue repair by differentiation into tissue-specific cell types or by production of trophic factors at the site of injury to stimulate tissue repair and/or to reduce self-inflicted damage mediated by the immune system (29). In PCa, BM-MSCs were also proven to be able to migrate to PCa and influence PCa cell growth (30), and invasion with increased PCa stem cell population (9).

We found, for the first time, that BM-MSCs, in addition to differentiating to CAFs or their direct function on PCa epithelial cells, they might also be able to promote the conversion of NFs to CAFs via secretion of TGF $\beta-1$. These results suggest that the recruited BM-MSCs may be able to transform into CAFs and further promote the conversion of normal fibroblasts to CAFs. The consequences of such increased CAFs may then enhance the PCa cell growth and invasion.

The significance of the above findings is not only adding new evidence of cross-talk between different cell types within the TME, it also provides a new potential target to suppress $\mathrm{PCa}$ progression. Targeting the infiltrating BM-MSCs via either interruption of interaction between PCa and BM-MSCs or preventing the conversion of NFs to CAFs via inhibition of TGF $\beta$-1 signal may result in suppression of the PCa progression.

\section{Acknowledgements}

We thank Karen Wolf for help with manuscript preparation. This study was supported by NIH grant CA156700 and George Whipple Professorship Endowment, Taiwan Department of Health Clinical Trial and Research Center of Excellence grant DOH99-TD-B-111-004 and China 973 grant CB518304.

\section{References}

1. Siegel R, Naishadham D and Jemal A: Cancer statistics, 2013. CA Cancer J Clin 63: 11-30, 2013.

2. Liao D, Luo Y, Markowitz D, Xiang R and Reisfeld RA: Cancer associated fibroblasts promote tumor growth and metastasis by modulating the tumor immune microenvironment in a 4T1 murine breast cancer model. PLoS One 4: e7965, 2009.

3. Zhu P, Baek SH, Bourk EM, Ohgi KA, Garcia-Bassets I, Sanjo H, Akira S, Kotol PF, Glass CK, Rosenfeld MG, et al: Macrophage/ cancer cell interactions mediate hormone resistance by a nuclear receptor derepression pathway. Cell 124: 615-629, 2006.

4. Zeng Y, Opeskin K, Goad J and Williams ED: Tumor-induced activation of lymphatic endothelial cells via vascular endothelial growth factor receptor- 2 is critical for prostate cancer lymphatic metastasis. Cancer Res 66: 9566-9575, 2006.

5. Gabbiani G, Ryan GB and Majne G: Presence of modified fibroblasts in granulation tissue and their possible role in wound contraction. Experientia 27: 549-550, 1971. 
6. Tomasek JJ, Gabbiani G, Hinz B, Chaponnier C and Brown RA Myofibroblasts and mechano-regulation of connective tissue remodelling. Nat Rev Mol Cell Biol 3: 349-363, 2002.

7. Franco OE, Shaw AK, Strand DW and Hayward SW: Cancer associated fibroblasts in cancer pathogenesis. Semin Cell Dev Biol 21: 33-39, 2010.

8. Zhao H and Peehl DM: Tumor-promoting phenotype of CD90hi prostate cancer-associated fibroblasts. Prostate 69: 991-1000, 2009.

9. Luo J, Ok Lee S, Liang L, Huang CK, Li L, Wen S and Chang C: Infiltrating bone marrow mesenchymal stem cells increase prostate cancer stem cell population and metastatic ability via secreting cytokines to suppress androgen receptor signaling. Oncogene 33: 2768-2778, 2014.

10. Huang CK, Tsai MY, Luo J, Kang HY, Lee SO and Chang C: Suppression of androgen receptor enhances the self-renewal of mesenchymal stem cells through elevated expression of EGFR. Biochim Biophys Acta 1833: 1222-1234, 2013.

11. Slavin S, Yeh CR, Da J, Yu S, Miyamoto H, Messing EM Guancial E and Yeh S: Estrogen receptor $\alpha$ in cancer-associated fibroblasts suppresses prostate cancer invasion via modulation of thrombospondin 2 and matrix metalloproteinase 3. Carcinogenesis 35: 1301-1309, 2014.

12. Tuxhorn JA, Ayala GE, Smith MJ, Smith VC, Dang TD and Rowley DR: Reactive stroma in human prostate cancer: induction of myofibroblast phenotype and extracellular matrix remodeling. Clin Cancer Res 8: 2912-2923, 2002.

13. Reinertsen T, Halgunset J, Viset T, Flatberg A, Haugsmoen LL and Skogseth H: Gene expressional changes in prostate fibroblasts from cancerous tissue. APMIS 120: 558-571, 2012.

14. Cirri P and Chiarugi P: Cancer associated fibroblasts: The dark side of the coin. Am J Cancer Res 1: 482-497, 2011.

15. Haubeiss S, Schmid JO, Mürdter TE, Sonnenberg M, Friedel G, van der Kuip H and Aulitzky WE: Dasatinib reverses cancerassociated fibroblasts (CAFs) from primary lung carcinomas to a phenotype comparable to that of normal fibroblasts. Mol Cancer 9: 168,2010

16. Ishii K, Mizokami A, Tsunoda T, Iguchi K, Kato M, Hori Y, Arima K, Namiki M and Sugimura Y: Heterogenous induction of carcinoma-associated fibroblast-like differentiation in normal human prostatic fibroblasts by co-culturing with prostate cancer cells. J Cell Biochem 112: 3604-3611, 2011.

17. Cirri P and Chiarugi P: Cancer-associated-fibroblasts and tumour cells: A diabolic liaison driving cancer progression. Cancer Metastasis Rev 31: 195-208, 2012.

18. Miyamoto H, Messing EM and Chang C: Androgen deprivation therapy for prostate cancer: Current status and future prospects. Prostate 61: 332-353, 2004.
19. Niu Y, Altuwaijri S, Lai KP, Wu CT, Ricke WA, Messing EM, Yao J, Yeh S and Chang C: Androgen receptor is a tumor suppressor and proliferator in prostate cancer. Proc Natl Acad Sci USA 105: 12182-12187, 2008

20. Lee SO, Tian J, Huang CK, Ma Z, Lai KP, Hsiao H, Jiang M, Yeh S and Chang C: Suppressor role of androgen receptor in proliferation of prostate basal epithelial and progenitor cells. J Endocrinol 213: 173-182, 2012.

21. Wang X, Lee SO, Xia S, Jiang Q, Luo J, Li L, Yeh S and Chang C: Endothelial cells enhance prostate cancer metastasis via IL- $6 \rightarrow$ androgen receptor $\rightarrow$ TGF- $\beta \rightarrow$ MMP-9 signals. Mol Cancer Ther 12: 1026-1037, 2013.

22. Izumi K, Fang LY, Mizokami A, Namiki M, Li L, Lin WJ and Chang C: Targeting the androgen receptor with siRNA promotes prostate cancer metastasis through enhanced macrophage recruitment via CCL2/CCR2-induced STAT3 activation. EMBO Mol Med 5: 1383-1401, 2013.

23. Gonda TA, Varro A, Wang TC and Tycko B: Molecular biology of cancer-associated fibroblasts: Can these cells be targeted in anti-cancer therapy? Semin Cell Dev Biol 21: 2-10, 2010.

24. Navab R, Strumpf D, Bandarchi B, Zhu CQ, Pintilie M, Ramnarine VR, Ibrahimov E, Radulovich N, Leung L, Barczyk M, et al: Prognostic gene-expression signature of carcinoma-associated fibroblasts in non-small cell lung cancer. Proc Natl Acad Sci USA 108: 7160-7165, 2011.

25. Zeisberg EM,Potenta S, Xie L, Zeisberg M and Kalluri R: Discovery of endothelial to mesenchymal transition as a source for carcinomaassociated fibroblasts. Cancer Res 67: 10123-10128, 2007.

26. Mishra PJ, Mishra PJ, Humeniuk R, Medina DJ, Alexe G, Mesirov JP, Ganesan S, Glod JW and Banerjee D: Carcinomaassociated fibroblast-like differentiation of human mesenchymal stem cells. Cancer Res 68: 4331-4339, 2008.

27. Direkze NC, Hodivala-Dilke K, Jeffery R, Hunt T, Poulsom R, Oukrif D, Alison MR and Wright NA: Bone marrow contribution to tumor-associated myofibroblasts and fibroblasts. Cancer Res 64: 8492-8495, 2004.

28. Comite P, Cobianchi L, Avanzini MA, Zonta S, Mantelli M, Achille V, De Martino M, Cansolino L, Ferrari C, Alessiani M, et al: Isolation and ex vivo expansion of bone marrow-derived porcine mesenchymal stromal cells: Potential for application in an experimental model of solid organ transplantation in large animals. Transplant Proc 42: 1341-1343, 2010.

29. Zhang H, Chen Z and Bie P: Bone marrow-derived mesenchymal stem cells as immunosuppressants in liver transplantation: A review of current data. Transfus Med Rev 26: 129-141, 2012.

30. Placencio VR, Li X, Sherrill TP, Fritz G and Bhowmick NA Bone marrow derived mesenchymal stem cells incorporate into the prostate during regrowth. PLoS One 5: e12920, 2010. 